\title{
A Case of Azathioprine Induced Severe Myelosuppression and Alopecia Totalis in IgA Nephropathy
}

\author{
Jae Choon Kim, M.D. \\ Ye Kyung Kim, M.D. \\ Hye Sun Hyun, M.D. \\ Eu Jin Park, M.D. \\ Hee Gyung Kang, M.D., Ph.D. \\ II Soo Ha, M.D., Ph.D. \\ Hae Il Cheong, M.D., Ph.D. \\ Departments of Pediatrics, Seoul \\ National University Children's Hospital, \\ Seoul, Korea \\ Corresponding author: \\ Hee Gyung Kang, M.D., Ph.D. \\ Department of Pediatrics, Seoul National \\ University Children's Hospital, 101 \\ Deahangno, Jongno-gu, Seoul, 03080 \\ Korea \\ Tel: +82-2-2072-0658 \\ Fax: +82-2-2072-0274 \\ E-mail:kanghg@snu.ac.kr \\ Received: 17 March 2017 \\ Revised: 30 March 2017 \\ Accepted: 31 March 2017
}

This is an open-access article distributed under the terms of the Creative Commons Attribution Non-Commercial License (http:// creativecommons.org/licenses/by-nc/4.0/) which permits unrestricted non-commercial use, distribution, and reproduction in any medium, provided the original work is properly cited.

Copyright (C) 2017The Korean Society of Pediatric Nephrology
Azathioprine is commonly used as immunosuppressive therapy for various inflammatory diseases including chronic glomerulonephritis. Myelosuppression is a common side effect of azathioprine, resulting in the need for dose reduction. However, severe pancytopenia or alopecia is not often encountered. Here, we report a case of severe myelosuppression, and alopecia totalis that occurred after azathioprine treatment in a patient with IgA nephropathy. A 10 -year-old boy with IgA nephropathy was treated with oral deflazacort and later with azathioprine. After 4 weeks, the patient complained of hair loss, and despite a dose reduction in azathioprine, he developed bone marrow suppression and alopecia totalis in two weeks. The blood indices and alopecia of the patient had returned to normal after azathioprine withdrawal and 3 consecutive doses of granulocyte colony-stimulating factor. We suggest that physicians remain vigilant to the side effects of azathioprine. Unusual hair loss after azathioprine treatment might suggest a defect in the metabolism of the drug, warranting the discontinuation of azathioprine to prevent more severe side effects.

Key words: Azathioprine, IgA nephropathy, Pancytopenia, Alopecia

\section{Introduction}

Azathioprine (AZA) is a purine analog that is widely used as an immunosuppressive drug in many medical conditions such as rheumatoid arthritis, inflammatory bowel disease, vasculitis, chronic hepatitis, glomerulonephritis and the prevention of rejection of solid organ transplants. It is a cytotoxic prodrug which interferes with DNA synthesis and inhibits the proliferation of rapidly growing cells. The most common adverse events of AZA are nausea, vomiting, malaise and myelosuppression ${ }^{1)}$. Less frequent events are hepatotoxicity, pancreatitis and alopecia. Up to $5-25 \%$ of patients who used AZA experienced side effects including severe myelosuppression, which might lead to life-threatening infection ${ }^{2}$. Thiopurine methyltransferase (TPMT) inactivates the toxic products of azathioprine metabolism, and the TPMT activity varies greatly between individuals ${ }^{3}$. Those with defective TPMT are at risk of suffering from the toxic effect of AZA. While many cases have been reported showing that severe bone marrow suppression as a side effect of AZA was associated with a deficiency in TPMT function in Europe ${ }^{4)}$, severe side effects in patients with normal TPMT function have been commonly 
reported in East Asia $^{5}$.

Here, we report a case of AZA-induced severe myelosuppression and alopecia in a child with IgA nephropathy (IgAN).

\section{Case report}

A 10-year-old boy presented with persistent proteinuria and hematuria detected by a school urine screening. Based on renal biopsy findings, the patient was diagnosed with IgA nephropathy Hass class IV. He was treated with deflazacort for two weeks, and then, AZA was given at an initial daily dosage of $25 \mathrm{mg}(0.8 \mathrm{mg} / \mathrm{kg})$ and increased to $50 \mathrm{mg}$ $(1.6 \mathrm{mg} / \mathrm{kg})$ per day two weeks after the initial visit. When the patient had taken AZA for 4 weeks, he started to complain of hair loss. Suspecting a side effect of the AZA, the dosage of the AZA was reduced to $25 \mathrm{mg}$ per day. Two weeks after the dose reduction (six weeks after he started AZA), the patient visited the emergency center with fever for two days. He also had a sore throat and mild cough. His vital signs were as follows: blood pressure, 101/59 $\mathrm{mmHg}$; pulse rate, 114 beats/min; respiratory rate, 20 breaths $/ \mathrm{min}$, and body temperature, $39.6^{\circ} \mathrm{C}$. On physical examination, alopecia totalis (Fig. 1) was observed as well as two slightly hypertrophic tonsils. His lung sound was clear without chest retraction or crackle. He had pancytopenia with a hemoglobin count of $8.4 \mathrm{~g} / \mathrm{dL}$, a white blood cell count of $640 / \mu \mathrm{L}$, a platelet count of $16 \mathrm{~K} / \mu \mathrm{L}$, and an absolute neutrophil count of $53 / \mu \mathrm{L}$. The serum concentration of Creactive protein was $6.01 \mathrm{mg} / \mathrm{dL}$ (reference, $0-0.5 \mathrm{mg} / \mathrm{dL}$ ). The urinalysis showed a positive result for albuminuria and hematuria with 50-99 erythrocytes/high power field (HPF) and an urine protein/creatinine ratio of $0.39(\mathrm{mg} / \mathrm{mg})$. Other laboratory results were unremarkable with a blood urea nitrogen of $8 \mathrm{mg} / \mathrm{dL}$, a serum creatinine of $0.56 \mathrm{mg} /$
dL, an AST of 14 IU/L, and an ALT of 11 IU/L. A bone marrow biopsy was not done.

Myelosuppression was not a typical symptom of IgAN and there were no other suspected medications (deflazacort, omega-3, enalapril) for a cause of myeosuppression except AZA. Suspecting a diagnosis of AZA induced neutropenia and complicating infection, the empirical antibiotics piperacillin and tazobactam were administered, and the AZA was discontinued. His fever subsided within two days, and no pathogen was identified in the blood culture or urine culture. Pancytopenia was managed with an injection of granulocyte colony stimulating factor and a transfusion of platelet and red blood cells. With the discontinuation of AZA, his hair loss was halted. He was discharged after 12 days of antibiotics treatment with a leu-

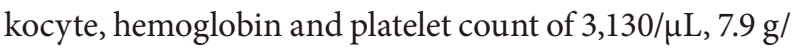
$\mathrm{dL}$ and $80 \mathrm{~K} / \mu \mathrm{L}$, respectively, (Fig. 2). Meanwhile, his proteinuria disappeared with a urine protein creatinine ratio of 0.13 (Table 1).

Later, a genetic analysis showed that the TPMT genotype was homozygote wild type. Now, the patient has been followed for one year at an outpatient clinic, and his laboratory findings have been normal including urinalysis taking only losartan.

\section{Discussion}

The patient in this case report was prescribed AZA for IgAN and experienced severe complications. AZA has been used in patients with severe IgAN, often along with steroids, as in our case. Reports on adverse events of AZA in patients with IgAN are few; on the other hand, there are many reports on myelosuppression after AZA in patients with other autoimmune diseases, such as inflammatory bowel disease and systemic lupus erythematosus $(\mathrm{SLE})^{6}$;

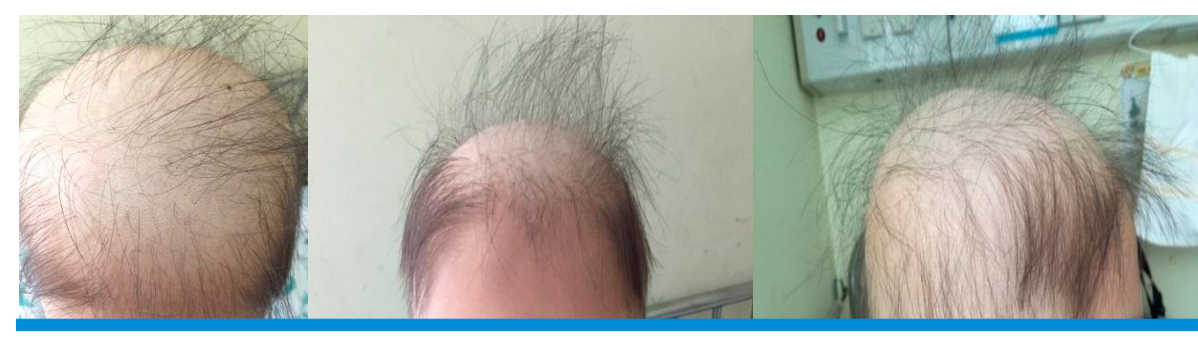

Fig. 1. Alopecia involving whole scalp after azathioprine treatment. 
Glinzler et al. reported that six out of 73 SLE patients receiving AZA developed leukopenia and required a dose reduction or discontinuation of the medication. Three of these patients had reversible pancytopenia, and two tolerated the reduced AZA dose. In patients with inflammatory bowel disease, pancytopenia was reported in $0.4-2 \%$ of the cases treated with $\mathrm{AZA}^{7}$. In our case, myelosuppression was detected 45 days after introduction of AZA. Similarly, myelosuppression has been reported to occur within 8 to 70 days after AZA is administered. Rarely, late myelosuppression has also been reported to occur within 11-27 weeks of AZA treatment or even in 11 years in one case ${ }^{8)}$. Our maximized AZA dose was $1.6 \mathrm{mg} / \mathrm{kg}$, which is a similar dose compared with other cases experiencing myelosuppression. Javier P. Gisbert et al. reported clinical reviews of thiopurine induced myelotoxicity in patients with inflammatory bowel disease. In this review, the author reviewed 66 studies (8,302 patients) and median AZA doses were reported from $1.3 \mathrm{mg} / \mathrm{kg}$ to $3 \mathrm{mg} / \mathrm{kg}^{9}$.

In our patient, myelosuppression was recovered 16 days after withdrawal of AZA. The time needed for normalization of myelosuppression after AZA withdrawal is variable ranging from 1 week to several month, regardless of genetic mutations ${ }^{10,11)}$. After oral administration, AZA is converted non-enzymatically to mercaptopurine (MP) and then undergoes a complex metabolism by three competitive enzymes:; xanthine oxidase metabolizes MP into 6-thioruic acid; TPMT metabolizes MP into inactive 6-methylmercaptopurine, and hypoxanthine-guanine phosphoribosyltransferase anabolizes MP into 6-thioguanine (6-TGN),

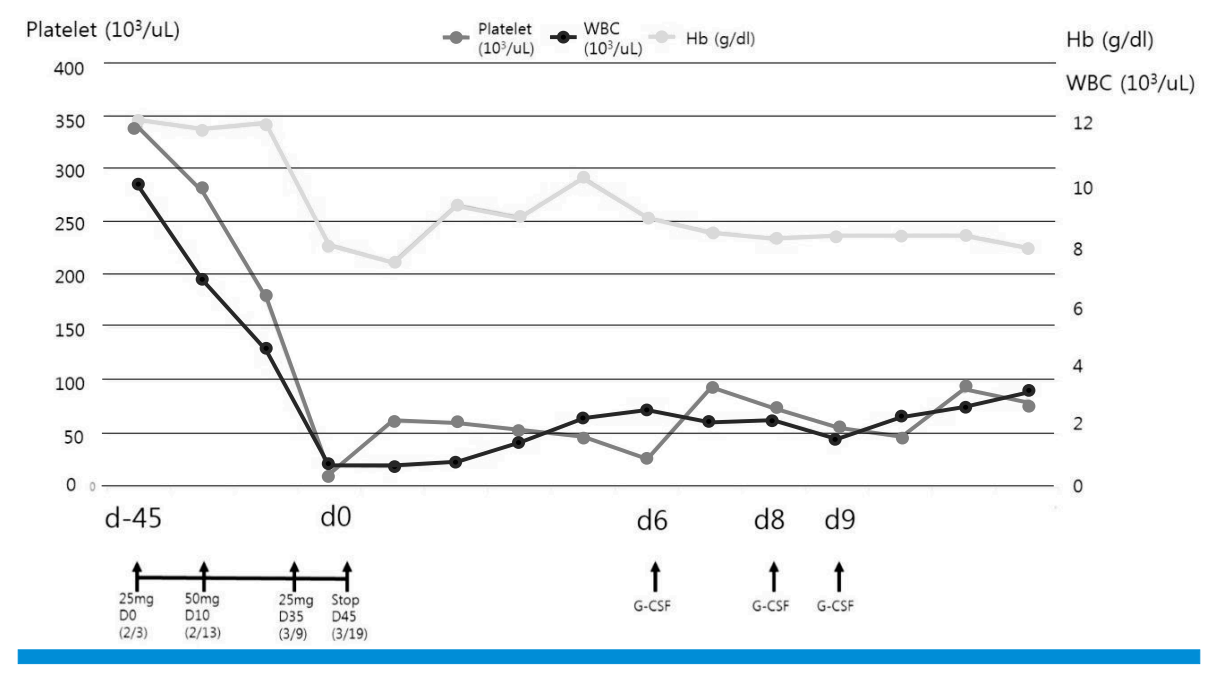

Fig. 2. Time course of the patient's WBC, hemoglobin and platelet levels in relation to the start and discontinuation of azathioprine (AZA) treatment.

Table 1. Laboratory Finding During Hospital Stay

\begin{tabular}{|c|c|c|c|c|c|c|c|c|c|}
\hline \multirow{2}{*}{ Parameter } & \multicolumn{9}{|c|}{ During hospital stay } \\
\hline & Day $-59^{*}$ & Day $-45^{\dagger}$ & Day $-35^{\ddagger}$ & Day $1^{\S}$ & Day 3 & Day 6 & Day $8^{\prime \prime}$ & Day $11^{\natural}$ & Day 16 \\
\hline Hemoglobin (g/dL) & 11.4 & 12.1 & 11.8 & 8.4 & 9.3 & 8.9 & 8.3 & 7.9 & 9.7 \\
\hline White blood cell (/uL) & 4,960 & 9,940 & 6,860 & 640 & 840 & 2,530 & 1,610 & 3,130 & 3,540 \\
\hline Absolute neutrophil count (/uL) & 2,579 & 7,187 & 3,903 & 77 & 8 & 0 & 349 & 551 & 1,429 \\
\hline Platelet(x103/uL) & 243 & 339 & 279 & 16 & 61 & 27 & 56 & 80 & 108 \\
\hline Urine protein/creatinine ratio & 0.28 & 1.39 & 0.56 & 0.39 & 0.68 & 0.13 & & & 0.22 \\
\hline Urine red blood cell (/HPF) & $>100$ & $>100$ & $30-49$ & 50-99 & & 10-19 & $>100$ & & $1-4$ \\
\hline
\end{tabular}

*The day steroid therapy was started.

${ }^{\dagger}$ The day AZA (25 mg per day) was started.

${ }^{*}$ The day dose of AZA (50 mg per day) was inceased.

${ }^{\S}$ At the time of admission due to neutropenic fever.

"Days filgrastim 225 mcg were given.

"At the time of discharge. 
which is responsible for the cytotoxic and immunosuppressive properties ${ }^{12}$. Therefore, TPMT is an important enzyme that reduces the cytotoxicity of AZA to inactive metabolites. If TPMT activity is reduced, MP would preferentially be metabolized into 6-TGN. In fact, a decrease in the TPMT activity is known as a predisposition to bone marrow suppression ${ }^{12)}$ reported in Europe and Middle east Asia ${ }^{4,13)}$. The US Food and Drug Administration (FDA) recommends genetic testing for TPMT before using azathioprine. Patients heterozygous for a nonfunctional TPMT allele are recommended AZA with a reduced dose. A drastically reduced dose, or alternative nonthiopurine immunosuppressant therapy is recommended for patients homozygous for nonfunctional TPMT alleles ${ }^{14)}$. On the other hand, in East Asia, TPMT levels have been reported to be normal in most patients who experienced myelosuppression after AZA, as in our case.

Recently, AZA-induced early leukopenia in Korean and Japanese studies has been reported to be associated with the Nudix hydrolase 15 (NUDT15) c.415C $>$ G variant ${ }^{15,16)}$. While the direct role of the NUDT15 gene in thiopurine metabolism is uncertain, the knockdown of the NUDT15 gene significantly promoted the degradation of proliferating cell nuclear antigen and inhibited DNA synthesis and cell cycle progression ${ }^{17)}$. Interestingly, all three patients with the NUDT15 c.415C > G variants reported by Lee YJ et al. suffered from AZA-induced leukopenia and alopecia totalis ${ }^{5}$. Unfortunately, NUDT15 genotyping was not done in our case. Furthermore, since studies on NUDT15 genotyping were performed to patients who had already experienced myelosuppression, there is no consensus on dose reduction after NUDT15 genotyping ${ }^{5,16)}$.

While AZA has been used for a long time as well as widely in various diseases, severe side effects as seen in our case are not rare. Therefore, careful monitoring of blood cell counts after AZA administration is advised. Interestingly, in East Asia, the NUDT15 variation seems more relevant than the TPMT variation in Europe, and severe hair loss was reported in the NUDT15 c. $415 \mathrm{C}>\mathrm{G}$ variants. Thus, the unusual hair loss after AZA administration might suggest a defect in NUDT15 or other metabolizers of AZA warranting the discontinuation of AZA. If available, genotyping before prescribing AZA might be helpful to prevent severe side effects.

\section{Acknowledgement}

This study was supported by a grant (HI12C0014) from the Korean Health Technology Research \& Development Project, Ministry of Health \& Welfare, Republic of Korea.

\section{Conflicts of interest}

No potential conflict of interest relevant to this article was reported.

\section{References}

1. Derijks L, Gilissen L, Hooymans P, Hommes D. Review article: thiopurines in inflammatory bowel disease. Alimentary pharmacology \& therapeutics 2006;24:715-29.

2. Mertens $H$, Hertel $G$, Reuther P, Ricker K. Effect of immunosuppressive drugs (azathioprine). Annals of the New York Academy of Sciences 1981;377:691-9.

3. Anstey A, Lennard L, Mayou S, Kirby J. Pancytopenia related to azathioprine--an enzyme deficiency caused by a common genetic polymorphism: a review. Journal of the Royal Society of Medicine 1992;85:752.

4. Collie-Duguid E, Sludden J, Li T, McLeod H. The frequency and distribution of thiopurine methyltransferase alleles in Caucasian and Asian populations. Pharmacogenetics and Genomics 1999; 9:37-42.

5. Lee YJ, Hwang EH, Park JH, Shin JH, Kang B, Kim SY. NUDT15 variant is the most common variant associated with thiopurineinduced early leukopenia and alopecia in Korean pediatric patients with Crohn's disease. Eur J Gastroenterol Hepatol 2016;28: 475-8.

6. Ginzler E, Sharon E, Diamond H, Kaplan D. Long-term maintenance therapy with azathioprine in systemic lupus erythematosus. Arthritis \& Rheumatology 1975;18:27-34.

7. Teml A, Schaeffeler E, Herrlinger KR, Klotz U, Schwab M. Thiopurine treatment in inflammatory bowel disease. Clinical pharmacokinetics 2007;46:187-208.

8. Lennard L, Van Loon JA, Weinshilboum RM. Pharmacogenetics of acute azathioprine toxicity: relationship to thiopurine methyltransferase genetic polymorphism. Clinical Pharmacology \& Therapeutics 1989;46:149-54.

9. Gisbert JP, Gomollón F. Thiopurine-induced myelotoxicity in patients with inflammatory bowel disease: a review. The American journal of gastroenterology 2008;103:1783-800.

10. Kirschner BS. Safety of azathioprine and 6-mercaptopurine in pediatric patients with inflammatory bowel disease. Gastroen- 
www.chikd.org

terology 1998;115:813-21.

11. O'Donoghue D, Dawson A, Powell-Tuck J, Bown R, Lennard-Jones J. Double-blind withdrawal trial of azathioprine as maintenance treatment for Crohn's disease. The Lancet 1978:312:955-7.

12. Gearry RB, Barclay ML. Azathioprine and 6-mercaptopurine pharmacogenetics and metabolite monitoring in inflammatory bowel disease. Journal of gastroenterology and hepatology 2005;20: 1149-57.

13. Gisbert JP, Luna M, Maté J, González-Guijarro L, Cara C, Pajares JM. Choice of azathioprine or 6-mercaptopurine dose based on thiopurine methyltransferase (TPMT) activity to avoid myelosuppression. A prospective study. Hepato-gastroenterology 2005;53:399-404.

14. DiPiero J, Teng K, Hicks JK. Should thiopurine methyltransferase
(TPMT) activity be determined before prescribing azathioprine, mercaptopurine, or thioguanine? Cleveland Clinic journal of medicine 2015;82:409-13.

15. Yang S-K, Hong M, Baek J, Choi H, Zhao W, Jung Y, et al. A common missense variant in NUDT15 confers susceptibility to thiopurine-induced leukopenia. Nature genetics 2014;46:1017-20.

16. Kakuta Y, Naito T, Onodera M, Kuroha M, Kimura T, Shiga H, et al. NUDT15 R139C causes thiopurine-induced early severe hair loss and leukopenia in Japanese patients with IBD. The Pharmacogenomics Journal 2016;16:280-5.

17. Yu Y, Cai J-P, Tu B, Wu L, Zhao Y, Liu X, et al. Proliferating cell nuclear antigen is protected from degradation by forming a complex with MutT Homolog2. Journal of Biological Chemistry 2009;284: 19310-20. 